Marquette University

e-Publications@Marquette

Winter 2017

\title{
Comparing Fixed-amount and Progressive-amount DRO Schedules for Tic Suppression in Youth with Chronic Tic Disorders
}

\author{
Matthew R. Capriotti \\ University of California, San Francisco \\ Jennifer E. Turkel \\ University of Wisconsin - Milwaukee \\ Rachel A. Johnson \\ University of Wisconsin - Milwaukee \\ Flint M. Espil \\ University of Wisconsin - Milwaukee \\ Douglas W. Woods \\ Marquette University, douglas.woods@marquette.edu
}

Follow this and additional works at: https://epublications.marquette.edu/psych_fac

Part of the Psychology Commons

\section{Recommended Citation}

Capriotti, Matthew R.; Turkel, Jennifer E.; Johnson, Rachel A.; Espil, Flint M.; and Woods, Douglas W., "Comparing Fixed-amount and Progressive-amount DRO Schedules for Tic Suppression in Youth with Chronic Tic Disorders" (2017). Psychology Faculty Research and Publications. 291.

https://epublications.marquette.edu/psych_fac/291 
Marquette University

e-Publications@Marquette

\section{Psychology Faculty Research and Publications/College of Arts and Sciences}

This paper is NOT THE PUBLISHED VERSION; but the author's final, peer-reviewed manuscript. The published version may be accessed by following the link in th citation below.

Journal of Applied Behavior Analysis, Vol. 50, No. 1 (Winter 2017): 106-120. DOI. This article is (C) Wiley and permission has been granted for this version to appear in e-Publications@Marquette. Wiley does not grant permission for this article to be further copied/distributed or hosted elsewhere without the express permission from Wiley.

\section{Comparing fixed-amount and progressive- amount DRO Schedules for tic suppression in youth with chronic tic disorders}

\section{Matthew R. Capriotti}

San José State University, University of California San Francisco, University of Wisconsin, Milwaukee, WI

Jennifer E. Turkel

University of Wisconsin, Milwaukee, WI

Rachel A. Johnson

University of Wisconsin, Milwaukee, WI

Flint M. Espil

Stanford University School of Medicine, University of Wisconsin, Milwaukee, WI

Douglas W. Woods

Marquette University, University of Wisconsin, Milwaukee, WI 


\section{Abstract}

Chronic tic disorders (CTDs) involve motor and/or vocal tics that often cause substantial distress and impairment. Differential reinforcement of other behavior (DRO) schedules of reinforcement produce robust, but incomplete, reductions in tic frequency in youth with CTDs; however, a more robust reduction may be needed to affect durable clinical change. Standard, fixed-amount DRO schedules have not commonly yielded such reductions, so we evaluated a novel, progressive-amount DRO schedule, based on its ability to facilitate sustained abstinence from functionally similar behaviors. Five youth with CTDs were exposed to periods of baseline, fixed-amount DRO (DRO-F), and progressive-amount DRO (DRO-P). Both DRO schedules produced decreases in tic rate and increases in intertic interval duration, but no systematic differences were seen between the two schedules on any dimension of tic occurrence. The DRO-F schedule was generally preferred to the DROP schedule. Possible procedural improvements and other future directions are discussed.

Chronic tic disorders (CTDs) are childhood-onset neurobehavioral disorders marked by the repeated presence of motor and/or vocal tics that persist for over one year (American Psychiatric Association, 2013). These conditions occur in up to $1.1 \%$ of youth, cause substantial functional impairment and distress, and frequently co-occur with other behavioral/psychiatric problems (Conelea, Woods, Zinner et al., 2011; Scharf, Miller, Mathews, \& BenShlomo, 2012).

Several efficacious (i.e., superior to placebo) treatments exist for pediatric CTDs, but each has significant shortcomings. Neuroleptic medications (e.g., risperidone, haloperidol, pimozide) decrease tic severity, but carry significant side effects (e.g., weight gain, slowed cognition, lethargy) that deter many from long-term use (Scahill et al., 2013). Nonpharmacological (i.e., behavioral) interventions produce decreases in tics comparable to medications, but without adverse side effects (Piacentini et al., 2010; Scahill et al., 2013; Wilhelm et al., 2012). Nevertheless, only about $50 \%$ of youth who receive behavioral interventions demonstrate a clinically significant treatment response (Piacentini et al., 2010), leaving half of patients substantially impaired by their tics after receiving behavior therapy. Such findings, although promising, call for further research on the refinement of behavioral treatment procedures.

Habit reversal training (HRT; Azrin \& Nunn, 1973), and an expanded version of HRT known as Comprehensive Behavioral Intervention for Tics (CBIT; Woods, Piacentini et al., 2008), is the most extensively researched behavioral intervention for CTDs. HRT begins with awareness training, during which the patient learns to reliably signal occurrences of the target tic (e.g., to raise a finger when a tic occurs, or when the urge to tic is experienced). Next, the patient is trained to engage in a competing response for one minute contingent on the presence of the urge to tic or the actual occurrence of the target tic. A competing response is a motor posture or, for vocal tics, a pattern of breathing that the patient can use to "block" tics from occurring. Finally, a social support component is implemented. Social support involves two elements. First, a caregiver provides verbal praise contingent on the child using the response to suppress tics. Second, the caregiver provides verbal prompts when the individual displays a tic but does not attempt to use the competing response. It is presumed that this praise and prompting will be sufficient to maintain competing response use outside of session. In turn, it is thought that repeated, sustained tic suppression via use of competing responses mediates long-term decreases in tic severity (Capriotti \& Woods, 2013; Himle, Woods, Piacentini, \& Walkup, 2006; Verdellen et al., 2008).

Unfortunately, it is not clear how regularly competing response use is actually reinforced in patients receiving HRT or CBIT. Published studies have not investigated the rate at which these putatively reinforcing outcomes (e.g., therapist praise, parental praise) occur during HRT (either in session or in the natural environment) and/or function as reinforcers (i.e., increase future occurrence of the competing response). Thus, it is plausible that many of the "treatment failures" observed in HRT and CBIT trials are due to inadequate reinforcement of 
sustained tic suppression (i.e., competing response use). Therefore, it may be possible to improve behavior therapy for CTDs by determining the schedule on which tic suppression should be reinforced in order to yield maximal therapeutic gains and treatment durability.

Many experiments have demonstrated that operant contingencies can affect tics (Capriotti, Brandt, Ricketts, Espil, \& Woods, 2012; Capriotti, Brandt, Turkel, Lee, \& Woods, 2014; Conelea, Woods, \& Brandt, 2011; Conelea \& Woods, 2008; Himle \& Woods, 2005; Himle, Woods, \& Bunaciu, 2008; Specht et al., 2013; Woods \& Himle, 2004; Woods, Walther, Bauer, Kemp, \& Conelea, 2009). These studies have employed resetting differential reinforcement of other behavior (DRO) schedules, in which tokens are provided following every $n$ second tic-free interval. Under these conditions, tic rates decrease reliably for a majority of subjects, though very few subjects exhibit zero or near-zero levels of tics. However, two considerations suggest that the procedures used in these studies may not be ideal for facilitating tic suppression for sustained periods. First, resetting DRO schedules operate as fixed-interval schedules, which engender "scalloped" patterns of responding (Ferster \& Skinner, 1957). Based on this, tics would presumably be relatively likely to occur shortly after reinforcer delivery. Indeed, Conelea, Bauer, Woods, and Kemp (2008, May) found that children with CTDs showed this pattern when exposed to DRO schedules of token reinforcement for tic suppression. Second, urges to tic may grow stronger throughout initial attempts at sustained tic suppression (Cotter, Capriotti, Brandt, \& Woods, 2011), thus increasing the value of competing reinforcement for tics (i.e., urge reduction), relative to reinforcers used in programmed reinforcement for tic suppression (i.e., tokens). According to the principle of momentary maximizing (i.e., that momentary reinforcer potency governs response allocation when multiple sources of reinforcement are available; Shimp, 1966; Todorov, Souza, \& Bori, 1993), tics would be expected to occur when urges are high and the next programmed reinforcer for tic suppression is relatively distant (i.e., early in the reinforcement interval). However, clinical behavior therapy for CTDs is thought to facilitate long-term tic reduction by providing new learning experiences wherein patients suppress tics in the presence of strong premonitory urges and sustain this suppression until the urge has dissipated (Capriotti \& Woods, 2013; Evers \& van de Wetering, 1994; Verdellen et al., 2008). Thus, although this pattern of alternating between tics and suppressing tics may be behaviorally efficient in a human operant setting, it appears unlikely to facilitate clinical change that persists in the absence of programmed contingencies.

One modification to DRO schedules involves increasing the magnitude of the programmed reinforcer during each consecutive interval that elapses without the occurrence of the target behavior. These "progressiveamount" schedules have been the subject of several studies within the area of substance use. In general, results have shown that progressive-amount schedules were more effective than fixed-amount schedules in engendering sustained abstinence from the target response of cigarette smoking or methamphetamine use (Roll \& Higgins, 2000; Roll, Higgins, \& Badger; 1996; Roll et al., 2006; Romanowich \& Lamb, 2015). It is important to note that researchers found comparable rates of submitting positive samples for fixed-amount and progressiveamount voucher schedules; the two schedules differed mainly in the extent to which they resulted in sustained drug-free periods.

Such findings have served as the basis for effective contingency management interventions for substance abuse (Dallery \& Glenn, 2005; Higgins, Silverman, Heil, \& Brady, 2007). Given several functional similarities between tics and substance abuse, it is possible that progressive-amount schedules may also enhance the facilitation of tic reduction. First, the motivating operations governing the two behaviors are quite similar. Just as the strength of premonitory urges increases during initial periods of sustained tic suppression, the strength of urges to engage in substance use also increases following initial periods of continuous abstinence (Budney, Moore, Vandrey, \& Hughes, 2003; McGregor et al., 2005). In both cases, engaging in the target behavior provides a brief reduction in the strength of the motivating operation (i.e., the urge). Second, both have been shown to be highly malleable by DRO techniques. Finally, a wealth of neurocognitive research suggests overlap in neurobiological 
factors underlying both tics and substance use (Blum et al., 1996; Singer et al., 2002; Wanat, Willuhn, Clark, \& Phillips, 2009). Considering these similarities between substance abuse and tics, it is reasonable to assume that schedules of reinforcement that are useful in promoting abstinence from substance use might also be useful in promoting increases in tic suppression.

In the present study, we compared progressive-amount DRO (DRO-P) and fixed-amount DRO (DRO-F) schedules of reinforcement for tic suppression. Tic rate and median intertic-interval duration (as an operationalization of sustained tic abstinence) were the primary dependent variables. We also evaluated subjects' relative preferences for the two DRO schedules and baseline conditions.

\section{METHOD}

\section{Subjects and Setting}

The study protocol was approved by the University of Wisconsin-Milwaukee Institutional Review Board. Six youth and their parent(s) were recruited (via print advertisements and patient flow through the University of Wisconsin-Milwaukee Tic Disorders Specialty Clinic), provided informed consent/assent, and initiated the screening process. All subjects met diagnostic criteria for Tourette syndrome. Table $\underline{1}$ contains data on other descriptive demographic and clinical characteristics of the subjects.

Table 1. Demographic and Clinical Background Data

\begin{tabular}{|l|l|l|l|l|}
\hline Name & Age & Gender & Comorbidity & Medications \\
\hline Alexa & $\begin{array}{l}16 \text { years, 5 } \\
\text { months }\end{array}$ & F & $\begin{array}{l}\text { ADHD, ODD, mood disorder } \\
\text { NOS }\end{array}$ & None \\
\hline Ben & $\begin{array}{l}10 \text { years, } 7 \\
\text { months }\end{array}$ & M & OCD & None \\
\hline Corey & 15 years & M & None & None \\
\hline David & 15 years & M & GAD, panic, OCD, ADHD & $\begin{array}{l}\text { Sertraline, atomoxetine, } \\
\text { methylphenidate }\end{array}$ \\
\hline
\end{tabular}

- Note. Demographic and clinical background data on included subjects. $\mathrm{F}=$ Female; $\mathrm{M}=$ Male; $A D H D=$ attention deficit hyperactivity disorder; ODD = oppositional defiant disorder; NOS = not otherwise specified; GAD = generalized anxiety disorder; panic = panic disorder.

Subjects were screened for eligibility according to the following criteria: (1) generally healthy males or females ages 9-17; (2) a diagnosis of Tourette Syndrome, Chronic Motor Tic Disorder, or Chronic Vocal Tic Disorder; (3) a Yale Global Tic Severity Score (YGTSS; Leckman et al., 1989) total tic severity score $\geq 14$ and $<35$ if diagnosed with Tourette syndrome or $\geq 10$ and $<20$ if diagnosed with CTD; (4) exhibits at least one tic per min during the initial 6-min baseline condition; (5) intellectual functioning in the low-average range or above as indicated by a score $\geq 75$ on the Wechsler Abbreviated Scale of Intelligence (WASI; Wechsler; 1999); (6) no significant suicidal ideation at present, reflected by a score $<9$ on the MINI-Kid suicidality index; (7) no history of behavioral treatment for tics; and (8) not currently taking a neuroleptic medication. Exclusion criteria were the presence of a psychotic disorder, substance use disorder, or autism spectrum disorder (parent-reported). Children on nonneuroleptic psychotropic medications were included if on a stable dosage for $\geq 6$ weeks. Children on stimulant medications were included as long as they had adhered to their medication regimen for the past 3 days and had not taken a dose within $10 \mathrm{hr}$ of the onset of the first experimental condition (if taking an extended-release formulation - within $6 \mathrm{hr}$ if taking an instant-release formulation). These criteria were designed to align with previous tic suppression studies and capture a subject population generally representative of youth who seek behavior therapy for CTDs. 


\section{Measures}

\section{Demographics form}

A parent-report measure was used to collect demographic information, treatment history, current medication status, and medical/psychiatric history for each subject.

Mini International Neuropsychiatric Interview for Children and Adolescents (MINI-Kid)

The MINI-Kid (Sheehan et al., 2010) is a brief structured diagnostic interview completed by the parent and child. This instrument is designed to assess for psychiatric disorders and has demonstrated favorable psychometric properties, including convergent validity with more time-consuming diagnostic interviews (Sheehan et al., 2010).

Wechsler Abbreviated Scale of Intelligence (WASI)

The WASI is a psychometrically acceptable measure of intellectual functioning for individuals aged 6-89 years (Wechsler, 1999). The two-subtest WASI was used to estimate full-scale IQ.

\section{Yale Global Tic Severity Scale (YGTSS)}

The YGTSS is a widely-utilized clinician-administered rating scale assessing tic severity. Separate scores are generated for motor and vocal tics (range: 0-25 each), based on ratings of tic number (of topographies), frequency, intensity (forcefulness), complexity, and interference (with other behavior), which are summed to yield a total tic severity score (range: 0-50). The YGTSS has good internal consistency, interrater reliability, and convergent and divergent validity (Leckman et al., 1989).

In-session tic data

Output files indicating the number and timing of tics throughout the experimental session served as the primary measure of tic occurrence. Measures of tic rate (number of tics per min) and median intertic interval (ITI) duration (in seconds) were derived from these primary records. We chose to evaluate median ITI duration because it better captures the dimension of behavior measured in prior studies of DRO-P schedules. In prior studies, time elapsed between instances of substance use was the measure of interest, rather than rate of substance use responses. For sessions in which fewer than two tics occurred, median ITI duration was recorded as $360 \mathrm{~s}$ (i.e., the duration of the session).

To allow for assessment of interobserver agreement (IOA), the head-on video stream of the subject during experimental sessions was recorded and later reviewed by a trained coder (i.e., secondary rater) who was blind to the hypotheses of this study. The coder viewed the video and recorded the occurrence of each tic by pressing a button on the keyboard, which generated a timestamp accurate to the nearest hundredth of a second. IOA coefficients were calculated using the block-by-block (i.e., proportional) method for each 10-s interval; overall IOA for each subject was computed as the mean of proportional agreement scores for all intervals across all sessions. IOA was not calculated for one subject (Ben) because his face and body were not consistently within view of the camera due to his failure to consistently comply with directions to face the camera directly. For this subject, the experimenter relied on the one-way mirror to monitor tics. For all other subjects, IOA was calculated for $100 \%$ of sessions. Mean IOA was $86 \%$ for Alexa (range, $78 \%-97 \%$ ), $80 \%$ for Corey (range, $57 \%-$ 97\%), and $86 \%$ for David (range, 56\%-100\%).

\section{Procedures and Experimental Design}

\section{Preexperimental assessment}

Subjects and their parents underwent an initial assessment to determine eligibility. After providing informed consent and assent, both the parent and child participated in interviews for the MINI-Kid and YGTSS. Children also completed the WASI, and parents completed the Demographics Form. A clinical psychology trainee (J.E.T.) administered, scored, and interpreted all measures, with ongoing supervision from the last author (D.W.W.), an 
expert in assessment and treatment of tics and comorbid conditions. Operational definitions of all tic topographies discussed during the YGTSS interview were generated for use in the experiment (see Supporting Information).

\section{Main experiment}

After completing the preexperimental assessment, subjects were exposed to eleven 6-min sessions in a multielement design. Previous research suggests 6 -min observations of tics yield response rates comparable to longer samples (e.g., 40 min; Himle, Chang, et al., 2006; Woods, Himle, et al., 2008). The experiment consisted of three baseline sessions (BL), four fixed-amount differential reinforcement of other behavior (DRO-F) sessions, and four progressive-amount differential reinforcement of other behavior (DRO-P) sessions. For each subject, the first session was BL. Each condition was associated with a unique background color on the experimental display. Prior to the first DRO session of either type, the subjects were told that every 1000 points they earned were worth $\$ 2$, although all subjects were paid $\$ 20$ postexperiment regardless of performance.

The present experiment employed the tic detector paradigm (Woods \& Himle, 2004) to measure tics using direct observation while controlling for individual differences in the influence of another individual's presence on tic rate (i.e., some individuals display tics more frequently when another is present, whereas others display them less frequently; Piacentini et al., 2006). At the beginning of the experiment, subjects were told the experimental apparatus was capable of detecting the occurrence of their tics. They were instructed to sit facing the apparatus with their hands away from their face during all experimental sessions, so that the "tic detector" could accurately monitor tics. Immediately prior to the onset of each session, the experimenter read instructions describing the contingencies in place for the upcoming session, conducted a presession manipulation check (detailed below), and exited the room. Sessions began shortly ( $<30 s)$ thereafter, as signaled by a tone audible to the subject. For the duration of the session, the words "TIC DETECTOR ON" appeared in the bottom right corner of the screen. Meanwhile, the experimenter monitored the subject via a one-way mirror and a live video feed from a webcam mounted on top of the apparatus' computer monitor. Upon the occurrence of a tic, the experimenter pressed a key on a keyboard connected to the computer supporting the experimental apparatus, which created a time-stamped record, and, when applicable, reset the DRO timer. Immediately following the offset of each session, the experimenter reentered the experimental room and conducted a postsession manipulation check (detailed below) to ensure that the subject had complied with the instructions given prior to session onset.

Instructions and manipulation check

Before each session, an experimenter read the subject instructions for the upcoming session and provided a description of the contingencies in operation. The experimenter asked the subject several yes/no questions about features of the programmed contingency to ensure basic understanding. If the subject answered these correctly, the experimenter acknowledged the correct responses, left the room, and the session began. In the event of incorrect responses, the experimenter first repeated the instructions and then re-presented the questions that had been answered incorrectly. This continued until the subject correctly responded to all questions relevant to the upcoming session.

At the end of each session, the experimenter asked the subject questions to evaluate his or her understanding of and compliance with the instructions for the just-terminated session. The experimenter recorded the subject's responses, but provided no feedback (see Supporting Information for the full text of pre- and postsession manipulation checks.). 


\section{Baseline}

During baseline (BL) sessions, no contingencies were programmed for tics, and no reference to points was displayed on the screen. Subjects were told that the tic detector would be on and counting their tics, but that no points would be available for stopping tics.

\section{DRO-F}

During DRO-F sessions, a fixed-amount, resetting DRO 10-s schedule of reinforcement was in effect; 30 points were delivered following each 10-s tic-free interval. Reinforcer delivery consisted of the point count incrementing by 30 and a brief tone sounding. The DRO timer was reset immediately upon the occurrence of a tic. Throughout the session, the text "Point Count:" followed by the number of points earned in the current session, was displayed in the center of the monitor. If a subject emitted no tics throughout the entire session, 1080 points would have been earned.

DRO-P

During DRO-P sessions, a progressive-amount, resetting 10-s DRO schedule of reinforcement was in effect. Reinforcer delivery, consisting of an incrementing of the point count and brief tone, occurred following each 10$s$ tic-free interval. Reinforcer magnitude was set at six points initially but increased by one point for each consecutive tic-free interval elapsed. In the event of a tic, the timer reset and the reinforcer magnitude reverted to six. From this point, the reinforcer magnitude was fixed at six points until three consecutive tic-free intervals elapsed. After this, the magnitude reset to the highest amount previously attained in that session and resumed incrementing by one point for each consecutive tic-free interval. The text "Point Count:" followed by the number of points earned in the current session was displayed in the center of the monitor. Following every third consecutive tic-free interval, a 20-point bonus was delivered along with the points programmed for that interval. When applicable, the availability of a bonus for completing the current interval without a tic was indicated on the screen. If subjects suppressed tics continuously throughout the session, they would have earned 1086 points in total. To equate potential rule governance across conditions, subjects were told that 1080 points were earnable.

\section{Preference probe}

Following the termination of the 11th session, subjects were instructed to select a final 15-min session from among the three types to which they were exposed (BL, DRO-F, and DRO-P). The experimenter explained the choice paradigm to the subject, asked the subject to "pick one," and left the room after the subject was assessed for comprehension of the experimental manipulation. Then, the subject was presented with three squares, each colored to match the screen color of one of the three experimental conditions. Additionally, each square contained a brief description of the contingencies that had been in effect during that condition. The subject made a choice by selecting a square using the arrows on the keyboard and pressing the enter key. The subject's selection was used as a behavioral index of preference. After the subject selected the condition, a 15min extinction session began, in which no programmed contingencies were in place, but the background was illuminated with the color previously correlated with the selected condition.

\section{Debriefing and reassent}

Following completion of all experimental activities, the experimenter debriefed subjects regarding the true nature of the experimental setup (i.e., that an experimenter was observing and recording tics). At this point, subjects reassented to have their data retained and analyzed. All subjects were paid $\$ 20$ regardless of performance. 
Independent variable integrity

Review of output files indicated that $100 \%$ of reinforcers were delivered within $1 \mathrm{~s}$ of their scheduled delivery time. All subjects passed all postsession manipulation checks with one exception: After the first BL session, Alexa indicated that the instructions were to suppress tics and that she had been attempting to do so. The experimenter provided feedback as to the correct instructions, and readministered the session. Only data from the readministration of this session are included in study analyses.

\section{RESULTS}

Figure $\underline{1}$ displays tic rate across sessions for each subject. For three of four subjects, tic rates were reliably lower during DRO-F and DRO-P sessions than during BL sessions. Tic rate did not differ substantially between DRO-F and DRO-P sessions for any subject. Additionally, the total numbers of reinforcers earned across DRO-F and DRO-P sessions were compared for each subject (Table 2). All subjects earned a higher number of reinforcers in DRO-F sessions than in DRO-P sessions.

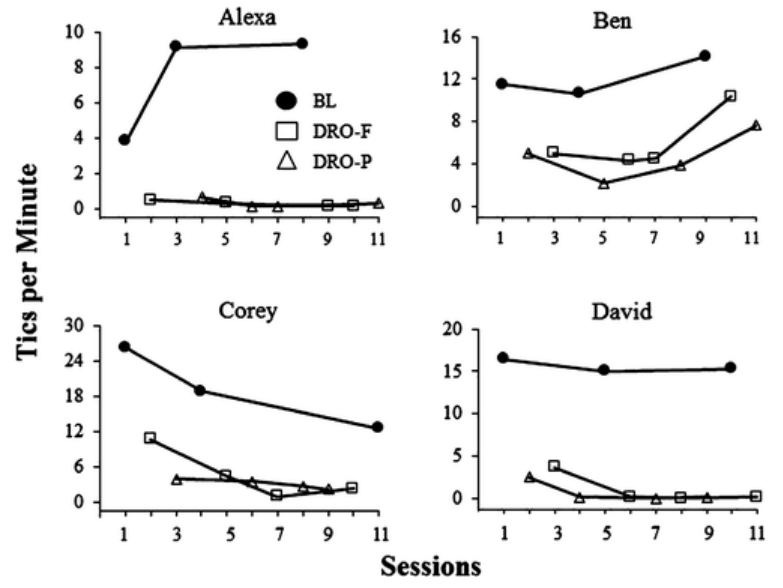

Figure 1 Tics per minute across sessions for each subject.

Table 2. Reinforcers Earned by Condition

\begin{tabular}{|l|l|l|}
\hline Subject ID & DRO-F & DRO-P \\
\hline 01 & 4140 & 3252 \\
\hline 03 & 2970 & 1212 \\
\hline 05 & 3450 & 1698 \\
\hline 06 & 4140 & 3400 \\
\hline
\end{tabular}

- Note. $\mathrm{DRO}-\mathrm{F}=$ fixed amount differential reinforcement of other behavior; $\mathrm{DRO}-\mathrm{P}=$ progressive amount differential reinforcement of other behavior.

Figure $\underline{2}$ shows the median intertic interval (ITI) duration observed across sessions for each subject. Median ITI duration was reliably higher during DRO-F and DRO-P sessions than during BL sessions for only one subject (Alexa) throughout the entire experiment. Data for the three other subjects showed that median ITI durations were generally higher during DRO sessions than during $\mathrm{BL}$, but the differences were less reliable. No subject showed reliable differences in median ITI duration between DRO-F and DRO-P sessions. 


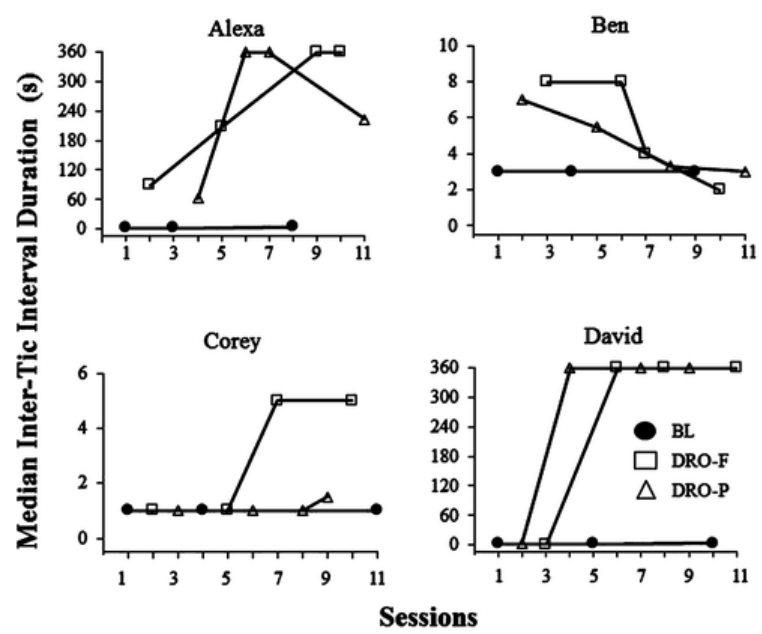

Figure 2 Median ITI durations across sessions for each subject.

The ITI duration results were inconsistent with previous research on substance use. This is surprising, given the robust decreases in tic rate observed in the present study. Therefore, we explored factors that may have contributed to these disparate findings. One possible reason is that we chose median ITI duration as a measure of "sustained abstinence" from tics. In contrast to this approach, previous studies in substance use examined the number of patients achieving sustained abstinence for the course of the study (Roll \& Higgins, 2000; Roll et al., 1996). Because the present study included very few instances in which subjects refrained from tics for an entire session, using that criterion for defining "sustained" tic suppression would have created inadequate range in the outcome variable. However, no prior studies were available to provide guidance on the duration at which tic suppression could be considered "sustained" (and clinically meaningful), versus relatively transient. Therefore, we conducted an additional analysis using a parametric approach to defining sustained tic suppression along various cutpoints $(t)$, with data combined across all DRO-F and DRO-P sessions. Sustained tic suppression was considered to occur throughout any tic-free period occurring $t$ seconds after the onset of a session or a subject's most recent tic. When a tic occurred, tseconds without a subsequent tic were required to pass until sustained tic suppression resumed.

Eleven duration-based cutpoints were established ideographically for Ben, Corey, and David. Because Alexa showed near-zero rates of tics during both DRO conditions, her data were not submitted to this parametric analysis. The first was the mean ITI duration observed across all BL sessions for that subject. Cutpoints two through ten were multiples of the mean BL ITI duration (2x-10x). The eleventh cutpoint was $60 \mathrm{~s}$, based on the duration of the competing response in HRT and CBIT (Azrin \& Nunn, 1973; Woods, Piacentini et al., 2008). Because sustained tic suppression began when an interval $t$ had elapsed, the total duration of tic suppression possible varied as a function of the cutpoint used (i.e., for a session wherein zero tics occurred, a 3-s cutpoint would yield $357 \mathrm{~s}$ of sustained suppression, whereas a 60-s cutpoint would yield $300 \mathrm{~s}$ of sustained suppression). To address this issue, we analyzed duration of sustained tic suppression as a proportion of the total duration possible (i.e., that would be yielded by complete tic abstinence) for each cutpoint.

Results of this analysis are shown in Figure $\underline{3}$. Ben and Corey tended to show greater sustained tic suppression for DRO-P than for DRO-F. By contrast, David generally showed greater sustained tic suppression under DRO-F than DRO-P. Alexa showed high levels of sustained tic suppression that were similar for the two DRO schedules. Results of this analysis provide additional evidence that the DRO-P schedule did not reliably engender more sustained tic suppression than the DRO-F schedule. 


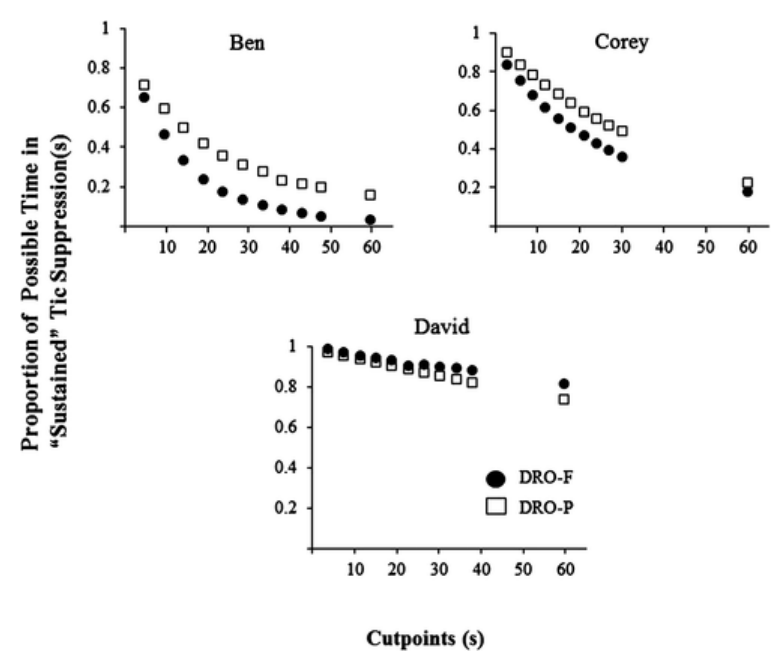

Figure 3 Proportion of possible time subjects engaged in tic suppression across cutpoints for each DRO schedule.

On the preference assessment trial, three of four subjects chose DRO-F and one (David) chose BL. No subject chose the DRO-P condition.

\section{DISCUSSION}

The current study compared fixed-amount and progressive-amount DRO schedules of reinforcement for tic suppression in youth with CTDs. Both DRO contingencies decreased tic rates to sub-baseline levels, but no systematic differences between the effects of DRO-P and DRO-F contingencies were observed. Median interticinterval durations generally increased during DRO sessions compared to $\mathrm{BL}$, but these differences were not reliable across all experimental sessions for most subjects. Post-hoc parametric analyses indicated no systematic differences in degree of sustained tic suppression observed under DRO-F and DRO-P schedules of reinforcement. Subjects earned fewer reinforcers under DRO-P than DRO-F, and the DRO-F schedule was generally preferred to DRO-P and to BL conditions.

The present results contrast with predictions based on previous research on progressive-amount schedules of reinforcement within the area of contingency management for substance use. Several possible explanations for this discrepancy are apparent. First, it is possible that the magnitude of reinforcement used in this study was too small to produce differences in responding between DRO schedules. Although the precise mechanisms through which DRO-P schedules reduce behavior are unclear, their efficacy is thought to hinge on the combination of the escalating nature of reinforcement for continued abstinence from the target behavior and the reinforcer magnitude reset contingency, which may function as response cost for engaging in the target behavior (Roll \& Higgins, 2000). If the discrepancy in value between the "progressed" reinforcer amount and the minimum amount to which the magnitude would reset in the event of a tic is not sufficient, the unique features of the DRO-P schedule may not, in fact, facilitate enhanced reductions of the target behavior.

It is also possible that the relative effects of DRO-F and DRO-P schedules depend on the nature of the target response and the subject's ability to detect its occurrence. Previous research showing greater reductions in behavior with DRO-P schedules than DRO-F schedules focused on cigarette smoking, a behavior of which subjects were aware (i.e., they could have indicated, in real time, when they were engaging in the target behavior and when they were not). Additionally, substance use involves a fairly complex response chain (e.g., taking a cigarette and lighter into one's hands, putting the cigarette in one's mouth, and lighting it). In contrast, tics have no necessary behavioral precursors, nor do they require ancillary materials. Many youth with CTDs cannot detect when their tics occur. Although no formal studies have evaluated the extent to which treatmentnaive patients are aware of their tics as they occur in real time, deficits in this area are common enough that 
awareness training has long been included as a formalized component of behavior therapy for CTDs (Azrin \& Nunn, 1973). Based on these considerations, one empirically-testable explanation for our failure to find differences is that the efficacy of DRO-P schedules may depend on individuals' awareness of the target response.

Additionally, it is possible that the effectiveness of DRO-P schedules may be moderated by certain other repertoires specific to the individual. For instance, DRO-P schedules may be more effective for individuals who discount delayed rewards less steeply (i.e., are more tolerant of delays). Within the context of the DRO-P used in the present study, subjects could obtain either an immediate reinforcer (urge reduction; Capriotti et al., 2014) for tics, or a more delayed reinforcer (points, the value of which increases incrementally as the delay increases). Along these lines, the effects of DRO-P schedules may also be age- and/or experience-dependent, because delay tolerance is known to increase throughout childhood and adolescence as prefrontal cortical structures, which subserve capacity to tolerate delays, mature (Green, Fry, \& Myerson, 1994).

Interestingly, DRO-P was superior to DRO-F with regard to efficiency (i.e., degree of behavior change per unit of reinforcement delivered). That is, DRO-P produced comparable rate-decreasing effects, but fewer reinforcers were delivered. Within the procedural context of this study, there were no pragmatic problems with delivering a greater number of reinforcers. However, in other contexts, this may present an issue. For instance, delivering many edible reinforcers in applied settings may lead to satiation throughout a session, decreasing the efficacy of that reinforcer. Also, when using activity-based reinforcers, session duration is increased with greater frequency of reinforcer delivery. Thus, DRO-P schedules may ultimately be useful in cases in which maximizing treatment gains per reinforcer delivery is of particular import.

Although the two DRO schedules had very similar effects on tics, we found differences in subjects' preferences for the two DRO schedules. On the choice trial, three of four subjects selected DRO-F, one selected BL, and none selected DRO-P. This indicates that, although presumably effortful, reinforced tic suppression was more preferred than the simulated "no treatment" (i.e., BL) condition. Two possible reasons for DRO-P being less preferred than DRO-F are apparent. For one, each subject earned substantially more reinforcers during DRO-F sessions than DRO-P sessions. An additional possibility is that the resetting reinforcer magnitude feature of the DRO-P schedule may have been aversive. Thus, the present findings suggest that treatment acceptability may sometimes be a concern when using progressive-amount schedules. However, due to the multitude of differences between this study and previous experiments on progressive-amount schedules (see below), only tentative conclusions can be made.

The present study was subject to methodological limitations that should be considered in interpreting its results. First, subjects were exposed to an interspersion of the three conditions within the context of a multielement design. This design, although useful for rapidly comparing multiple experimental conditions, leaves open the possibility that multiple treatment interference may have influenced outcomes; that is, exposure to one DRO schedule may have influenced responding under the other DRO schedule. Therefore, it is possible that repeated exposure to each DRO schedule alone (e.g., in the context of a withdrawal design, or via a between-groups design) would have yielded differentiation between the two schedules.

The preference assessment was also subject to certain limitations. We used a single trial to assess preference. Thus, the present findings suggest the schedule that was most preferred; repeated pairwise selections provide a more compelling result (Hanley, 2010). It is also unknown whether the three subjects who chose DRO-F in the choice trial would have preferred DRO-P to BL. Future research in this area should incorporate repeated measures and continuance of the assessment after determination of the most preferred context.

The discrepancy in total reinforcers earned across conditions is also an important factor to consider when interpreting the results of the present study. The total number of reinforcers earnable for perfect tic 
suppression was equated across conditions, but due to imperfect tic suppression, fewer reinforcers were delivered in DRO-P sessions than in DRO-F sessions. Comparable effects on tic rate were seen across schedules, suggesting that DRO-P schedules engendered more efficient tic suppression in terms of effects per reinforcer delivered. However, given the absence of empirical data on reinforcer magnitude and tic suppression, it is difficult to draw strong inferences about the implications of these differences.

Other limitations were associated with our approach to determining the duration of the DRO interval. We set the DRO interval duration a priori, based on the average rate of tics seen in previous experiments in this line of research (i.e., $\sim 6$ tics per min at baseline). We did not individualize the DRO interval duration for each subject based on the distributions of interresponse interval observed under baseline conditions, as is often done when DRO schedules are used for treatments in typical settings. This decision was consistent with the approach used in previous tic suppression studies. That said, it is possible that individualizing the DRO for each subject according to his or her baseline data would have altered outcomes of this study (e.g., because the effort associated with suppressing tics for $10 \mathrm{~s}$ may vary as a function of baseline tic rate). For instance, an ideographic approach would have yielded a shorter DRO interval for Ben, which could have led to more robust decreases in tic rate during DRO sessions. In future work, it may be advisable to set the initial DRO interval based on baseline data and systematically increase its duration across sessions (e.g., Repp, Deitz, \& Speir, 1974).

The present findings contribute to the nascent literature on progressive-amount schedules of reinforcement. As discussed above, this report extends the use of these schedules to target behaviors outside of substance use and provides preliminary data on their acceptability. Future research could aim to elucidate variables that govern performance under, and preference for, progressive-amount schedules. Investigations related to the contributions of both subject-specific factors (e.g., age, delay tolerance) and parameters of the DRO-P contingency (e.g., the amount-resetting feature, reinforcer magnitude, DRO interval) may be of particular interest. The interaction between baseline response rate and the effects of DRO-P schedules is of particular interest, as subjects with high baseline response rates may contact fewer reinforcing elements of the DRO-P schedule (Romanowich \& Lamb, 2015).

This study also contributes to the behavioral literature on tic disorders. Present findings support the generality of tic suppression to schedules of positive reinforcement beyond the fixed-amount DRO schedules used in previous research. Additionally, this was the first study to formally evaluate the social validity and patient acceptability of reinforced tic suppression procedures, and present results provide an initial indication that youth with CTDs prefer to engage in reinforced tic suppression rather than a no-suppression baseline. Although the results of the present study do not strongly recommend DRO-P schedules as a superior alternative to DRO-F schedules for tic suppression, the importance of research aimed at developing improved techniques for facilitating efficient tic suppression remains high. The present investigation focused on the use of high-density reinforcement schedules to promote sustained tic suppression in a contrived setting, because research suggests this practice may lead to decreases in tic severity when reinforcement is withdrawn (Verdellen et al., 2008). To be clear, this is not to suggest that the kinds of high-density schedules described in this study would be implemented long-term in children's typical environments. Rather, time-limited practice in a contrived setting is explored as a potential adjunct to other interventions (e.g., HRT/CBIT), which are feasible for use in typical environments.

This project demonstrates how a translational approach can be used to test these kinds of potential modifications before bringing them to scale in clinical trials. Research on behavior therapy for CTDs broadly, and tic suppression-based treatments specifically, is burgeoning, and continued investigation holds the promise to yield more effective and accessible treatments for individuals affected by CTDs. 
Supporting Information

\begin{tabular}{|l|l|}
\hline Filename & Description \\
\hline jaba360-sup-0001-Appendix.docxWord 2007 & $\begin{array}{l}\text { Appendix S1. Instructions for Experimental Conditions } \\
\text { and Manipulation Checks } \\
\text { document , 23.7 KB }\end{array}$ \\
\hline
\end{tabular}

Please note: The publisher is not responsible for the content or functionality of any supporting information supplied by the authors. Any queries (other than missing content) should be directed to the corresponding author for the article.

\section{References}

American Psychiatric Association ( 2013). Diagnostic and statistical manual of mental disorders ( $5^{\text {th }}$ ed.). Washington, DC: American Psychiatric Association. doi:10.1176/appi.books.9780890423349

Azrin, N. H., \& Nunn, R. G. ( 1973). Habit-reversal: A method of eliminating nervous habits and tics. Behaviour Research and Therapy,11, 619-628. doi: 10.1016/0005-7967(73)90119-8

Blum, K., Sheridan, P. J., Wood, R. C., Braverman, E. R., Chen, T. K., Cull, J. G., \& Comings, D. E. ( 1996). The $D_{2}$ dopamine receptor gene as a determinant of reward deficiency syndrome. Journal of the Royal Society of Medicine, 89, 396- 400. doi: 10.1177/014107689608900711

Budney, A. J., Moore, B. A., Vandrey, R. G., \& Hughes, J. R. ( 2003). The time course and significance of cannabis withdrawal. Journal of Abnormal Psychology, 112, 393- 402.

Capriotti, M. R., Brandt, B. C., Ricketts, E. J., Espil, F. M., \& Woods, D. W. ( 2012). Comparing the effects reinforcement and response-cost contingencies on tic suppression. Journal of Applied Behavior Analysis,45, 251- 263. doi: 10.1901/jaba.2012.45-251

Capriotti, M. R., Brandt, B.C., Turkel, J. E., Lee, H.-J., \& Woods, D. W. ( 2014). Negative reinforcement and premonitory urges in Tourette syndrome: An experimental evaluation. Behavior Modification, 38,276- 296. doi: 10.1177/0145445514531015

Capriotti, M. R., \& Woods, D. W. ( 2013). Cognitive-Behavioural therapy. In D. Martino \& J. Leckman (Eds.), Tourette Syndrome New York, NY: Oxford University Press.

Conelea, C. A., Bauer, C., Woods, D. W., \& Kemp, J. ( 2008, May).Exploring the impact of reinforced suppression on the temporal distribution of tics. Poster presented at the annual meeting of the Association for Behavior Analysis International. Chicago, IL.

Conelea, C. A., \& Woods, D. W. ( 2008). The influence of contextual factors on tic expression in Tourette syndrome: a review. Journal of Psychosomatic Research, 65, 487-496. doi:10.1016/j.jpsychores.2008.04.010

Conelea, C. A., Woods, D. W., \& Brandt, B. C. ( 2011). The impact of a stress induction task on tic frequencies in youth with Tourette syndrome. Behaviour Research and Therapy, 49, 492- 497. doi:10.1016/j.brat.2011.05.006

Conelea, C. A., Woods, D. W., Zinner, S. H., Budman, C., Murphy, T., . . \& Walkup, J. ( 2011). Exploring the impact of chronic tic disorders on youth: Results from the Tourette Syndrome Impact Survey. Child Psychiatry \& Human Development, 42, 219- 42. doi:10.1007/s10578-010-0211-4

Cotter, S. P., Capriotti, M. R., Brandt, B. C.\& Woods, D. W. ( 2011, November). Habituation to premonitory urges in Tourette syndrome: A controlled laboratory evaluation. Poster presented at the Tic and Impulse Control Disorders Special Interest Group meeting at the annual meeting of the Association for Behavioral and Cognitive Therapies, Toronto, Canada.

Dallery, J., \& Glenn, I. M. ( 2005). Effects of an internet-based voucher reinforcement program for smoking abstinence: A feasibility study. Journal of Applied Behavior Analysis, 38, 349- 357. doi: $10.1901 /$ jaba.2007.40-1 
Evers, R. A. F., \& van de Wetering, B. J. M. ( 1994). A treatment model for motor tics based on a specific tensionreduction technique. Journal of Behavior Therapy and Experimental Psychiatry, 25 , 255- 260. doi: 10.1016/0005-7916(94)90026-4

Ferster, C. B., \& Skinner, B. F. ( 1957). Schedules of reinforcement. New York: Appleton-Century-Crofts.

Green, L., Fry, A. F., \& Myerson, J. ( 1994). Discounting of delayed rewards: A life-span comparison. Psychological Science, 5, 33- 36. doi: 10.1111/j.1467-9280.1994.tb00610.x

Hanley, G. P. ( 2010). Toward effective and preferred programming: A case for the objective measurement of social validity with recipients of behavior-change programs. Behavior Analysis in Practice,3,13-21.

Higgins, S. T., Silverman, K., Heil, S. H., \& Brady, J. V. ( 2007).Contingency management in substance abuse treatment. New York, NY: The Guilford Press.

Himle, M. B., Chang, S., Woods, D. W., Pearlman, A., Buzzella, B., Bunaciu, L., \& Piacentini, J. C. (2006). Establishing the feasibility of direct observation in the assessment of tics in children with chronic tic disorders. Journal of Applied Behavior Analysis, 39, 429- 440. doi:10.1901/jaba.2006.63-06

Himle, M. B., \& Woods, D. W. ( 2005). An experimental evaluation of tic suppression and the tic rebound effect. Behaviour Research \& Therapy , 43, 1443-1451. doi: 10.1016/i.brat.2004.11.002

Himle, M. B., Woods, D. W., \& Bunaciu, L. ( 2008). Evaluating the role of the contingency in differentially reinforced tic suppression. Journal of Applied Behavior Analysis, 41, 285- 289. doi: 10.1901/jaba.2008.41-285

Himle, M. B., Woods, D. W., Piacentini, J. C., \& Walkup, J. T. ( 2006).Brief review of habit reversal training for Tourette syndrome. Journal of Child Neurology , 21 , 719- 725. doi: $10.1177 / 08830738060210080101$

Leckman, J. F., Riddle, M. A., Hardin, M. T., Ort, S. I., Swartz, K. L., Stevenson, J., \& Cohen, D. J. ( 1989). The Yale Global Tic Severity Scale: Initial testing of a clinician-rated scale of tic severity. Journal of the American Academy of Child \& Adolescent Psychiatry , 28, 566- 573. doi:10.1097/00004583-198907000-00015

McGregor, C., Srisurapanont, M., Jittiwutikarn, J., Laobhripatr, S., Wongtan, T., \& White, J. M. ( 2005). The nature, time course and severity of methamphetamine withdrawal. Addiction, 100, 1320-1329. doi:10.1111/i.1360-0443.2005.01160.x

Piacentini, J., Himle, M. B., Chang, S., Baruch, D. E., Buzzella, B. A., Pearlman, A., \& Woods, D. W. ( 2006). Reactivity of tic observation procedures to situation and setting. Journal of Abnormal Child Psychology, 34, 647- 656. doi:10.1007/s10802-006-9048-5

Piacentini, J., Woods, D. W., Scahill, L., Wilhelm, S., Peterson, A. L., Chang, S., . . Walkup, J. ( 2010). Behavior therapy for children with Tourette disorder: A randomized controlled trial. JAMA: Journal of the American Medical Association, 303, 1929- 1937. doi:10.1001/jama.2010.607

Repp, A. C., Deitz, S. M., \& Speir, N. C. ( 1974). Reducing stereotypic responding of retarded persons by the differential reinforcement of other behavior. American Journal of Mental Deficiency, 79, 279- 284.

Roll, J. M., \& Higgins, S. T. ( 2000). A within-subject comparison of three different schedules of reinforcement of drug abstinence using cigarette smoking as an exemplar. Drug and Alcohol Dependence, 58,103- 109. doi:10.1016/S0376-8716(99)00073-3

Roll, J. M., Higgins, S. T., \& Badger, G. J. ( 1996). An experimental comparison of three different schedules of reinforcement of drug abstinence using cigarette smoking as an exemplar. Journal of Applied Behavior Analysis, 29, 495- 505. doi:10.1901/iaba.1996.29-495

Roll, J. M., Huber, A., Sodano, R., Chudzynski, J. E., Moynier, E., \& Shoptaw, S. ( 2006). A comparison of five reinforcement schedules for use in contingency management-based treatment of methamphetamine abuse. The Psychological Record, 56, 67-81.

Romanowich, P. R., \& Lamb, R. J. ( 2015). The effects of fixed versus escalating reinforcement schedules on smoking abstinence. Journal of Applied Behavior Analysis , 48, 25- 37. doi: 10.1002/jaba.185

Scahill, L., Woods, D.W., Himle, M.B., Peterson, A.L., Wilhelm, S., Piacentini, J. C., . . Mink, J. W. ( 2013). Current controversies on the role of behavior therapy in Tourette syndrome. Movement Disorders,28, 1179-1183. doi:10.1002/mds.25488 
Scharf, J.M., Miller, L.L., Mathews, C.A., \& Ben-Shlomo, Y. ( 2012)Prevalence of Tourette syndrome and chronic tics in the population-based Avon longitudinal study of parents and children cohort. Journal of American Academy of Child \& Adolescent Psychiatry, 51, 192- 201. doi:10.1016/i.aac.2011.11.004

Sheehan, D. V., Sheehan, K. H., Shytle, R. D., Janavs, J., Bannon, Y., Rogers, J. E., . . . Wilkinson, B. ( 2010). Reliability and validity of the mini international neuropsychiatric interview for children and adolescents (MINI-KID). Journal of Clinical Psychiatry, 71, 313- 326. doi:10.4088/JCP.09m05305whi

Shimp, C. P. ( 1966). Probabilistically reinforced choice behavior in pigeons. Journal of the Experimental Analysis of Behavior, 9, 443-455. doi:10.1901/jeab.1966.9.443

Singer, H. S., Szymanski, S., Giuliano, J., Yokoi, F., Dogan, A. S., Brasic, J. R., . . Wong, D. F. ( 2002). Elevated intrasynaptic dopamine release in Tourette's syndrome measured by PET. American Journal of Psychiatry, 159, 1329- 1336.

Specht, M. W., Woods, D. W., Nicotra, C. M., Kelly, L. M., Ricketts, E. J., Conelea, C. A., \& Walkup, J. T. ( 2013). Effects of tic suppression: Ability to suppress, rebound, negative reinforcement, and habituation to the urge versus extinction learning. Behaviour Research and Therapy, 51, 24- 30. doi:10.1016/i.brat.2012.09.009

Todorov, J.C., Souza, D.G., \& Bori, C.M. ( 1993). Momentary maximizing in concurrent schedules with a minimum interchangeover interval. Journal of the Experimental Analysis of Behavior, 60, 415- 435. doi:10.1901/jeab.1993.60-415

Verdellen, C. W. J., Hoogduin, C. A. L., Kato, B. S., Keijsers, G. P. J., Cath, D. C., \& Hoijtink, H. B. ( 2008). Habituation of premonitory sensations during exposure and response prevention treatment in Tourette's syndrome. Behavior Modification, 32, 215- 227. doi: $10.1177 / 0145445507309020$

Wanat, M. J., Willuhn, I., Clark, J. J., \& Phillips, P. E. M. ( 2009). Phasic dopamine release in appetitive behaviors and drug abuse. Current Drug Abuse Reviews, 2, 195- 213. doi:10.2174/1874473710902020195

Wechsler, D. ( 1999). Wechsler Abbreviated Scale of Intelligence. San Antonio, TX: The Psychological Corporation.

Wilhelm, S., Peterson, A. L., Piacentini, J. C., Woods, D. W., Deckersbach, T., Sukhodolsky, D. G., ... Scahill, L. ( 2012). Randomized trial of behavior therapy for adults with Tourette syndrome. Archives of General Psychiatry, 69, 795- 803. doi: 10.1001/archgenpsychiatry.2011.1528

Woods, D.W., \& Himle, M. B. ( 2004). Creating tic suppression: Comparing the effects of verbal instruction to differential reinforcement. Journal of Applied Behavior Analysis , 37, 417- 420. doi:10.1901/jaba.2004.37-417

Woods, D. W., Himle, M. B., Miltenberger, R. G., Carr, J. E., Osmon, D. C., Karsten, A. M., . . Bosch, A. (2008). Durability, negative impact, and neuropsychological predictors of tic suppression in children with chronic tic disorders. Journal of Abnormal Child Psychology, 36 ,237-245. doi:10.1007/s10802-0079173-9

Woods, D. W., Piacentini, J. C., Chang, S. W., Deckersbach, T., Ginsberg, G. S., Peterson, A. L., ...Wilhelm, S. (2008). Managing Tourette syndrome: A behavioral intervention for children and adults, therapist guide. New York, NY: Oxford University Press.

Woods, D. W., Walther, M. R., Bauer, C. C., Kemp, J. J., \& Conelea, C. A.( 2009). The development of stimulus control over tics: A potential explanation for contextually-based variability in the symptoms of Tourette syndrome. Behaviour Research and Therapy, 47, 41-47. doi:10.1016/i.brat.2008.10.013 